\title{
Association of Elevated Serum Soluble Cd226 Levels With the Disease Activity and Flares of Systemic Lupus Erythematosus
}

\section{Miki Nakano}

Kyushu University: Kyushu Daigaku

Masahiro Ayano ( $\square$ ayano.masahiro.811@m.kyushu-u.ac.jp)

Kyushu University Graduate School of Medical Sciences https://orcid.org/0000-0002-2954-9676

\section{Kazuo Kushimoto}

Kyushu University: Kyushu Daigaku

\section{Shotaro Kawano}

Kyushu University: Kyushu Daigaku

\section{Kazuhiko Higashioka}

Kyushu University: Kyushu Daigaku

\section{Shoichiro Inokuchi}

Kyushu University: Kyushu Daigaku

Hiroki Mitoma

Kyushu University: Kyushu Daigaku

Yasutaka Kimoto

Kyushu University Beppu Hospital: Kyushu Daigaku Byoin Beppu Byoin

Mitsuteru Akahoshi

Kyushu University: Kyushu Daigaku

Nobuyuki Ono

Kyushu University: Kyushu Daigaku

\section{Yojiro Arinobu}

Kyushu University: Kyushu Daigaku

\section{Koichi Akashi}

Kyushu University: Kyushu Daigaku

\section{Takahiko Horiuchi}

Kyushu University Beppu Hospital: Kyushu Daigaku Byoin Beppu Byoin

\section{Hiroaki Niiro}

Kyushu University: Kyushu Daigaku 
Keywords: Systemic lupus erythematosus, Soluble CD226, Disease activity, Flare, NPSLE, Biomarker Posted Date: April 6th, 2021

DOI: https://doi.org/10.21203/rs.3.rs-377056/v1

License: (c) (1) This work is licensed under a Creative Commons Attribution 4.0 International License. Read Full License 


\section{Abstract}

Background: CD226 is an activating receptor expressed on the cell surface of natural killer cells and T cells. A soluble form of CD226 (sCD226) is known to be shed from the membrane type of CD226 (mCD226). Although CD226 polymorphism and mCD226 are known to be involved in systemic lupus erythematosus (SLE), the involvement of SCD226 in SLE is still unknown. Therefore, we aimed to reveal the association of SCD226 with SLE.

Methods: We measured serum SCD226 levels using an enzyme-linked immunosorbent assay in 58 SLE patients and 33 healthy controls (HCs) and evaluated their associations with SLE Disease Activity Index 2000 (SLEDAI-2K), clinical manifestations, and laboratory data. We defined the maximum values of sCD226 in HCs as a cut-off level and compared the cumulative probability of flare for patients with high and low sCD226 levels.

Results: Serum sCD226 levels showed no significant differences between SLE patients and HCs. However, SCD226 levels were significantly elevated in active SLE patients with a SLEDAI-2K score of $\geq 20$ compared with HCs. In SLE patients, sCD226 levels were significantly correlated with SLEDAI-2K scores and anti-dsDNA antibody titers. Moreover, the cumulative probability of flare was markedly higher in patients with high SCD226 than in those with low SCD226. In patients with neuropsychiatric involvement, sCD226 levels were elevated and reflected neuropsychiatric disease activity.

Conclusion: Serum SCD226 levels were associated with disease activity and flares of SLE. Thus, it may be a useful biomarker for SLE, and its monitoring allows for more precise SLE management.

\section{Background}

Systemic lupus erythematosus (SLE) is a multi-systemic autoimmune disease with diverse clinical manifestations [1, 2], commonly with renal and neuropsychiatric involvement, and shows variable severities [2,3]. Because the clinical course of SLE varies and flares occur several times, it is important to diagnose clinical manifestations and monitor the disease activity [4]. Biomarkers are valuable for assessing disease activities and predicting flares, but useful biomarkers have not been established yet.

The pathogenesis of SLE is multifactorial and includes genetic factors [2, 5-7]. Genome-wide association studies have reported an association between nonsynonymous rs763361 polymorphism in CD226 and SLE in multiple ancestries [8-11]. CD226 is a transmembrane glycoprotein mainly expressed on T cells and natural killer (NK) cells, which acts as an activating receptor and mediates cytotoxicity [12-14]. CD226 plays an important role in the immune system, and a previous study showed that the proportion of CD226 on NK cells was decreased in active SLE patients and that CD $226^{+}$NK cells may be involved in the immunopathogenesis of SLE [15].

A soluble form of CD226 (sCD226), which is shed from the membrane type of CD226 (mCD226) in human serum, has been identified. The utility of SCD226 as a biomarker has been reported in acute graft- 
versus-host disease (aGVHD) $[16,17]$ and some types of cancers [18-20]. As for autoimmune diseases, a more recent study found that serum SCD226 levels were associated with disease activity in rheumatoid arthritis (RA) [21]. Although several findings suggest that CD226 is involved in the pathogenesis of SLE $[8-11,15]$, the association between SCD226 and SLE is still unknown.

This study aimed to reveal the association of SCD226 with SLE by measuring serum SCD226 levels using an enzyme-linked immunosorbent assay (ELISA) in SLE patients, as well as by evaluating the associations between SCD226 levels and the disease activity, clinical manifestations, and flares of SLE.

\section{Methods}

\section{Study population}

We studied 58 Japanese patients who were treated for SLE at the Kyushu University Hospital between 2014 and 2020. We enrolled patients who met at least four of the American College of Rheumatology revised criteria for SLE [22] and had no other autoimmune disease, infection, or cancer. Many of these patients were treated with corticosteroids, hydroxychloroquine, and immunosuppressive drugs, either as monotherapy or in combination. Among these 58 SLE patients, we were able to assess 11 patients both before and after treatment. We studied 33 healthy controls (HCs) as well.

This study was approved by the ethics committee of Kyushu University Hospital (approval number 2019481) in accordance with the Helsinki Declaration. All participants gave written informed consent.

\section{Data collection}

We obtained the following information from the medical records of the patients: demographic data, clinical manifestations, laboratory findings, and medications at baseline and after treatment. Disease activity was evaluated using the SLE Disease Activity Index 2000 (SLEDAI-2K) [23], with active SLE defined as having a SLEDAI-2K score of $\geq 20$ [24]. Clinical manifestations were classified using the British Isles Lupus Assessment Group (BILAG) 2004 index [25], and patients with BILAG category A or category B at the time of SCD226 level measurement were defined as those with each clinical manifestation. Neuropsychiatric disease activity was assessed by BILAG 2004 index [25]. A flare was defined as a measurable increase in disease activity usually leading to a change of treatment $[4,26]$.

\section{Enzyme-linked immunosorbent assay}

Serum SCD226 levels were measured via sandwich ELISA, in accordance with a previous report [16]. In summary, 96-well plates were coated with purified anti-human CD226 (DNAM-1) antibody (TX25;

BioLegend, San Diego, CA, USA) $(8 \mu \mathrm{g} / \mathrm{ml}, 100 \mu \mathrm{l} /$ well) for 2 hours at room temperature and then washed with washing buffer (0.05\% Tween 20). The plates were blocked using a blocking buffer ( $1 \%$ BSA in PBS, $100 \mu \mathrm{l} /$ well) for 2 hours at room temperature and then washed. Recombinant human DNAM-1/CD226 Fc chimera protein (as a standard) (R\&D Systems, Minneapolis, MN, USA) and serum samples were added at $100 \mu \mathrm{l} /$ well and incubated overnight at $4^{\circ} \mathrm{C}$. The plates were washed and then incubated with human 
DNAM-1/CD226 biotinylated antibody (R\&D Systems) $(0.6 \mu \mathrm{g} / \mathrm{ml}, 100 \mu \mathrm{l} /$ well) for 1 hour at room temperature. After washing, streptavidin-horseradish peroxidase (R\&D Systems) (1:200 in a washing buffer, $100 \mu \mathrm{l} /$ well) was added and incubated for 30 minutes at room temperature. The plates were washed and then reacted with 3,3',5,5'-tetramethylbenzidine substrate reagent set (BD Biosciences, San Jose, CA, USA) (100 $\mu \mathrm{l} /$ well) for 20 minutes at room temperature. The reaction was stopped by $\mathrm{H}_{2} \mathrm{SO}_{4}$ (2N) $(50 \mu \mathrm{l} /$ well), and then absorbance was measured at $450 \mathrm{~nm}$ using a microtiter plate reader (Thermo Fisher Scientific, Waltham, MA, USA). All values were determined in duplicate. The assay range was 0.1 to $20.0 \mathrm{ng} / \mathrm{ml}$.

\section{Statistical analysis}

Results are expressed as the median and interquartile range unless otherwise stated. Comparisons between two groups were done using the Student's t-test for normally distributed continuous variables or using the Mann-Whitney $U$ test for non-normally distributed variables. When making multiple group comparisons, the Steel test was used for non-normally distributed variables, setting HCs as a control. The correlations between two continuous variables were analyzed using Spearman's rank correlation. Flaring episodes were represented via the Kaplan-Meier method and compared using log-rank tests. All tests were two-tailed and $P$-values $<0.05$ were considered statistically significant. All analyses were performed using the JMP software, version 15 (SAS Institute, Cary, NC, USA).

\section{Results}

\section{Serum SCD226 levels are increased in active SLE patients and reflect disease activity}

To study the association between SCD226 and SLE, we first measured serum SCD226 levels using ELISA in 58 SLE patients (mean age, 41.0 years; 53 females) and 33 HCs (mean age, 36.2 years; 28 females). No significant differences were found between SLE patients and HCs in terms of age and gender. The baseline characteristics of the SLE patients are shown in Table 1. Although there was no significant difference between SLE patients and HCs regarding median levels of serum SCD226, these still had a wide range of values and the SLE patients had higher values than had HCs (Figure 1). Because of this, we classified the SLE patients into three groups on the basis of SLEDAI-2K scores, and then compared sCD226 levels between HCs and these groups. Serum SCD226 levels were found to be significantly elevated in active SLE patients than in other SLE patients and HCs (Figure 2A).

We next studied the relationship between SCD226 levels and SLE disease activity. Serum SCD226 levels had a significantly positive correlation with SLEDAI-2K $(\rho=0.39 ; P=0.003)$ (Figure 2B). When compared with conventional biomarkers, SCD226 levels were significantly correlated with anti-dsDNA antibody titers $(\rho=0.28 ; P=0.035)$ and inversely correlated with serum levels of C3 $(\rho=-0.17 ; P=0.19)$ and C4 $(\rho=$ $-0.20 ; P=0.14$ ) (Supplementary Figure S1).

Furthermore, we also examined SCD226 levels in 11 SLE patients before and after treatment. The changes in SCD226 had a significant correlation with the changes in SLEDAI-2K scores $(\rho=0.67 ; P=$ 
0.024) (Figure 2C).

\section{sCD226 levels can predict disease flare}

In this study, flares occurred in 15 out of 58 SLE patients examined. Because the highest sCD226 level in HCs was $10.0 \mathrm{ng} / \mathrm{ml}$, we defined this as the cut-off value for classifying SLE patients as having high and low sCD226 levels. The cumulative probability of flare for patients with high sCD226 (Figure 3, shown in solid lines) was significantly higher than that for those with low SCD226 (Figure 3, shown in dashed lines) $(P=0.016)$.

\section{sCD226levels are increased in SLE patients with neuropsychiatric manifestation andreflect neuropsychiatric disease activity}

We then assessed the association between SCD226 levels and clinical manifestations of SLE. Serum sCD226 levels were elevated in patients with mucocutaneous, hematological, musculoskeletal, and/or neuropsychiatric manifestations (Table 2).

Because neuropsychiatric systemic lupus erythematosus (NPSLE) is a major vital organ manifestation of SLE [3], we further studied the relationships between sCD226 levels and neuropsychiatric manifestation. Serum sCD226 levels were elevated in patients with active neuropsychiatric manifestation (Table 2). Serum sCD226 levels were also significantly higher in active NPSLE patients than in previous NPSLE patients (Figure 4A). Moreover, among active NPSLE patients, sCD226 levels were significantly higher in patients with BILAG category $A$, which is defined as severe disease activity, than in those with BILAG category B (Figure 4B).

\section{Discussion}

In this study, we demonstrated that serum sCD226 levels were significantly increased in active SLE patients and were associated with disease activity and neuropsychiatric manifestation. We also showed that SLE patients with high SCD226 levels had a high probability of experiencing disease flares.

CD226 is an immunoglobulin superfamily expressed on the cell membrane of NK cells, T cells, B cells, monocytes, and platelets [12-14]. A soluble form of CD226 has been identified and was reported to be shed from the cell membrane maybe by a certain protease [16]. CD226 is a costimulatory adhesion molecule involved in certain immune functions such as mediating cytotoxic signals [12-14], the ligands of which are CD112 and CD155 [13]. This costimulatory molecule has a paired receptor, T cell immunoreceptor with immunoglobulin and immunoreceptor tyrosine-based inhibitory motif domain (TIGIT), which is a coinhibitory receptor that inhibits the interaction between CD155 and CD226, in turn also inhibits the activation of T cells and NK cells $[27,28]$. On the basis of the results of genome-wide association studies and functional analyses of mCD226 on T cells or NK cells, CD226 is thought to play an important role in the pathogenesis of autoimmune diseases such as SLE [8-11, 15], RA [11, 29-32], 
and systemic sclerosis [33-35]. However, the utility of SCD226 as a biomarker and its functions in these diseases remain unknown.

In this study, we aimed to investigate the association between SCD226 and SLE, and found that serum sCD226 levels were significantly elevated in active SLE patients than in HCs and were associated with disease activity. Moreover, we also found that the cumulative probability of flare was significantly higher in patients with high SCD226 than in patients with low SCD226, indicating that SCD226 may be predictive of flares. According to the 2019 EULAR recommendation, SLE treatment should aim for remission or low disease activity and prevention of flares [4]. Although many useful biomarkers for monitoring disease activity have been reported [36, 37], no proper biomarkers for predicting flares were found. In our study, serum SCD226 levels were associated with not only disease activity but also with SLE flares. Therefore, sCD226 may be a useful biomarker for SLE.

In this study, we further investigated the association between SCD226 levels and neuropsychiatric manifestation, which is a frequent major organ manifestation in SLE [3, 4]. Diverse neuropsychiatric symptoms make it difficult to distinguish from other diseases and hinder a proper diagnosis [3]. Additionally, its management is challenging because of the variable severity [3]. Useful biomarkers for both diagnosing and monitoring disease activity are required to manage NPSLE properly, but none have been established yet $[38,39]$. Although cerebrospinal fluid (CSF) tests from lumbar puncture are valuable for the exclusion of infectious diseases and some CSF biomarkers, such as anti-ribosomal P protein antibodies, IgG index, and IL-6, may be useful for assessing disease activity, the procedure is invasive and difficult to repeat for monitoring $[3,38]$. In our study, serum SCD226 levels were elevated in patients with neuropsychiatric involvement and reflected neuropsychiatric disease activity. The findings indicate that serum SCD226 may be a useful biomarker for both diagnosing and monitoring neuropsychiatric manifestation of SLE.

Regarding the function of SCD226 in cancer, several studies reported that SCD226 may block the cytotoxicity of NK cells by blocking CD155 or CD112 [18], and that SCD226 could directly inhibit the proliferation of cancer cells in vitro [19]. In aGVHD, Kanaya et al. explained that binding of SCD226 to CD155 may cancel the inhibitory signals by TIGIT in T cells [16]. In mouse SLE models, treatment with TIGIT-Ig fusion protein reduced autoantibody production and improved survival rate [40]. These findings indicate the binding of SCD226 to CD155 may cancel the inhibitory signals by TIGIT in SLE as well; this interaction is likely involved in the pathogenesis of SLE. Although we showed the possible utility of SCD226 as a biomarker for SLE in this study, we did not study the immune functions of SCD226 in SLE. Therefore, further analyses are required to reveal the functions of SCD226 in SLE.

This study had some limitations. First, our study had a small sample size and was conducted at a single center. This study needs to be replicated with a larger sample size in a multicenter setting. Second, the functions of sCD226 are still unknown; further analyses are required to reveal this. Lastly, our study was a retrospective study. To ensure the association of SCD226 levels with the prognosis of SLE, a prospective study should be performed. 


\section{Conclusions}

In conclusion, we showed that serum SCD226 levels were elevated in active SLE patients and were associated with disease activity and prognosis. Serum SCD226 may be a useful biomarker for SLE, and its monitoring allows for more precise SLE management.

\section{Abbreviations}

SLE: Systemic lupus erythematosus; NK: natural killer; sCD226: soluble CD226; mCD226: membrane CD226; aGVHD: acute graft-versus-host disease; RA: rheumatoid arthritis; ELISA: enzyme-linked immunosorbent assay; HCs: healthy controls; SLEDAI-2K: SLE Disease Activity Index 2000; BILAG: British Isles Lupus Assessment Group; NPSLE: neuropsychiatric systemic lupus erythematosus; TIGIT: T cell immunoreceptor with immunoglobulin and immunoreceptor tyrosine-based inhibitory motif domain; CSF: cerebrospinal fluid.

\section{Declarations}

\section{Ethics approval and consent to participate}

This study was approved by the ethics committee of Kyushu University Hospital (approval number 2019481) in accordance with the Helsinki Declaration. All participants gave written informed consent.

\section{Consent for publication}

Not applicable.

\section{Availability of data and materials}

All data generated or analyzed during this study are included in this published article and its supplementary information files.

\section{Competing interests}

The authors declare that they have no competing interests.

\section{Funding}

This work was supported by Japan Society for the Promotion of Science [grant number JSPS KAKENHI 19K17887].

\section{Authors' contributions}

$\mathrm{MN}$ and MAy participated in study conception and design. MN, MAy, KK, SK, KH, and SI participated in data acquisition and analysis. MN, MAy, KK, HM, YK, MAK, NO, YA, KA, TH, and HN contributed to the 
interpretation of results. MN was a major contributor in writing the manuscript. All authors read and approved the final manuscript.

\section{Acknowledgements}

The authors thank Enago for the English language review.

\section{References}

1. Tsokos GC. Systemic lupus erythematosus. N Engl J Med. 2011;365(22):2110-21.

2. Tsokos GC. Autoimmunity and organ damage in systemic lupus erythematosus. Nat Immunol. 2020;21(6):605-14.

3. Schwartz N, Stock AD, Putterman C. Neuropsychiatric lupus: new mechanistic insights and future treatment directions. Nat Rev Rheumatol. 2019;15(3):137-52.

4. Fanouriakis A, Kostopoulou M, Alunno A, Aringer M, Bajema I, Boletis JN, et al. 2019 Update of the EULAR recommendations for the management of systemic lupus erythematosus. Ann Rheum Dis. 2019;78(6):736-45.

5. Tsokos GC, Lo MS, Reis PC, Sullivan KE. New insights into the immunopathogenesis of systemic lupus erythematosus. Nat Rev Rheumatol. 2016;12(12):716-30.

6. Harley ITW, Kaufman KM, Langefeld CD, Harley JB, Kelly JA. Genetic susceptibility to SLE: New insights from fine mapping and genome-wide association studies. Nat Rev Genet. 2009;10(5):28590.

7. Bentham J, Morris DL, Cunninghame Graham DS, Pinder CL, Tombleson P, Behrens TW, et al. Genetic association analyses implicate aberrant regulation of innate and adaptive immunity genes in the pathogenesis of systemic lupus erythematosus. Nat Genet. 2015;47(12):1457-64.

8. Sun C, Molineros JE, Looger LL, Zhou XJ, Kim K, Okada Y, et al. High-density genotyping of immunerelated loci identifies new SLE risk variants in individuals with Asian ancestry. Nat Genet. 2016;48(3):323-30.

9. Nie D, Li H, Yan G, Wang Z, He Z, Zhou W. Gene-gene interaction between CD40 and CD226 gene on systemic lupus erythematosus in the Chinese Han population. Rheumatol Int. 2016;36(12):1657-62.

10. Wang YF, Zhang Y, Zhu Z, Wang TY, Morris DL, Shen JJ, et al. Identification of ST3AGL4, MFHAS1, CSNK2A2 and CD226 as loci associated with systemic lupus erythematosus (SLE) and evaluation of SLE genetics in drug repositioning. Ann Rheum Dis. 2018;77(7):1078-84.

11. Maiti AK, Kim-Howard X, Viswanathan P, Guillén L, Qian X, Rojas-Villarraga A, et al. Non-synonymous variant (Gly307Ser) in CD226 is associated with susceptibility to multiple autoimmune diseases. Rheumatology. 2010;49(7):1239-44.

12. Shibuya A, Campbell D, Hannum C, Yssel H, Franz-Bacon K, McClanashan T, et al. DNAM-1, a novel adhesion molecule involved in the cytolytic function of T lymphocytes. Immunity. 1996;4(6):573-81. 
13. Xu Z, Jin B. A novel interface consisting of homologous immunoglobulin superfamily members with multiple functions. Cell Mol Immunol. 2010;7(1):11-9.

14. Martinet L, Smyth MJ. Balancing natural killer cell activation through paired receptors. Nat Rev Immunol. 2015;15(4):243-54.

15. Huang Z, Fu B, Zheng SG, Li X, Sun R, Tian Z, et al. Involvement of CD226 ${ }^{+}$NK Cells in Immunopathogenesis of Systemic Lupus Erythematosus. J Immunol. 2011;186(6):3421-31.

16. Kanaya M, Shibuya K, Hirochika R, Kanemoto M, Ohashi K, Okada M, et al. Soluble DNAM-1, as a predictive biomarker for acute Graft-Versus-Host disease. PLoS One. 2016;11(6):1-12.

17. Goshima Y, Nakaoka S, Ohashi K, Sakamaki H, Shibuya K, Shibuya A. A mathematical model for dynamics of soluble form of DNAM-1 as a biomarker for graft-versus-host disease. PLoS One. 2020;15(2):1-12.

18. Xu Z, Zhang T, Zhuang R, Zhang Y, Jia W, Song C, et al. Increased levels of soluble CD226 in sera accompanied by decreased membrane CD226 expression on peripheral blood mononuclear cells from cancer patients. BMC Immunol. 2009;10:1-8.

19. Hou S, Zheng X, Wei H, Tian Z, Sun R. Recombinant soluble CD226 protein directly inhibits cancer cell proliferation in vitro. Int Immunopharmacol. 2014;19(1):119-26.

20. Takahashi N, Sugaya M, Suga H, Oka T, Kawaguchi M, Miyagaki T, et al. Increased Soluble CD226 in Sera of Patients with Cutaneous T-Cell Lymphoma Mediates Cytotoxic Activity against Tumor Cells via CD155. J Invest Dermatol. 2017;137(8):1766-73.

21. Mosaad YM, El-Toraby EE, Tawhid ZM, Abdelsalam Al, Enin AF, Hasson AM, et al. Association between CD226 polymorphism and soluble levels in rheumatoid arthritis: Relationship with clinical activity.Immunol Invest. 2018; 47:264-278.

22. Hochberg MC. Updating the American College of Rheumatology revised criteria for the classification of systemic lupus erythematosus. Arthritis Rheum. 1997;40:1725.

23. Gladman DD, Ibañez D, Urowitz MB. Systemic Lupus Erythematosus Disease Activity Index 2000. J Rheumatol. 2002;29(2):288-91.

24. Cook RJ, Gladman DD, Pericak D, Urowitz MB. Prediction of short term mortality in systemic lupus erythematosus with time dependent measures of disease activity. J Rheumatol. 2000;27(8):1892-5.

25. Isenberg DA, Rahman A, Allen E, Farewell V, Akil M, Bruce IN, et al. BILAG 2004. Development and initial validation of an updated version of the British Isles Lupus Assessment Group's disease activity index for patients with systemic lupus erythematosus. Rheumatology. 2005;44(7):902-6.

26. Ruperto N, Hanrahan LM, Alarcón GS, Belmont HM, Brey RL, Brunetta P, et al. International consensus for a definition of disease flare in lupus. Lupus. 2011;20(5):453-62.

27. Yu X, Harden K, Gonzalez LC, Francesco M, Chiang E, Irving B, et al. The surface protein TIGIT suppresses $\mathrm{T}$ cell activation by promoting the generation of mature immunoregulatory dendritic cells. Nat Immunol. 2009;10(1):48-57. 
28. Stanietsky N, Simic H, Arapovic J, Toporik A, Levy O, Novik A, et al. The interaction of TIGIT with PVR and PVRL2 inhibits human NK cell cytotoxicity. Proc Natl Acad Sci USA. 2009;106(42):17858-63.

29. Hafler JP, Maier LM, Cooper JD, Plagnol V, Hinks A, Simmonds MJ, et al. CD226 Gly307Ser association with multiple autoimmune diseases. Genes Immun. 2009;10(1):5-10.

30. Suzuki T, Ikari K, Kawaguchi Y, Yano K, Iwamoto T, Kawamoto M, et al. Non-synonymous variant (Gly307Ser) in CD226 is associated with susceptibility in Japanese rheumatoid arthritis patients. Mod Rheumatol. 2013;23(1):200-2.

31. Lee YH, Bae SC, Song GG. Association between the CTLA-4, CD226, FAS polymorphisms and rheumatoid arthritis susceptibility: A meta-analysis. Hum Immunol. 2015;76(2-3):83-9.

32. Fasth AER, Björkström NK, Anthoni M, Malmberg KJ, Malmström V. Activating NK-cell receptors costimulate $\mathrm{CD}^{+}{ }^{+} \mathrm{CD}_{28}{ }^{-} \mathrm{T}$ cells in patients with rheumatoid arthritis. Eur J Immunol. 2010;40(2):37887.

33. Dieudé P, Guedj M, Truchetet ME, Wipff J, Revillod L, Riemekasten G, et al. Association of the CD226 Ser307 variant with systemic sclerosis. Arthritis Rheum. 2011;63(4):1097-105.

34. Jin J, Chou C, Lima M, Zhou D, Zhou X. Systemic Sclerosis is a Complex Disease Associated Mainly with Immune Regulatory and Inflammatory Genes. Open Rheumatol J. 2014;8(1):29-42.

35. Ayano M, Tsukamoto H, Kohno K, Ueda N, Tanaka A, Mitoma H, et al. Increased CD226 Expression on $\mathrm{CD}^{+} \mathrm{T}$ Cells Is Associated with Upregulated Cytokine Production and Endothelial Cell Injury in Patients with Systemic Sclerosis. J Immunol. 2015;195(3):892-900.

36. Arriens C, Wren JD, Munroe ME, Mohan C. Systemic lupus erythematosus biomarkers: the challenging quest. Rheumatology. 2017;56:i32-45.

37. Capecchi R, Puxeddu I, Pratesi F, Migliorini P. New biomarkers in SLE: from bench to bedside. Rheumatology. 2020. doi:10.1093/rheumatology/keaa484.

38. Jeltsch-David H, Muller S. Neuropsychiatric systemic lupus erythematosus: Pathogenesis and biomarkers. Nat Rev Neurol. 2014;10(10):579-96.

39. Hanly JG, Kozora E, Beyea SD, Birnbaum J. Review. Nervous System Disease in Systemic Lupus Erythematosus: Current Status and Future Directions. Arthritis Rheumatol. 2019;71(1):33-42.

40. Liu S, Sun L, Wang C, Cui Y, Ling Y, Li T, et al. Treatment of murine lupus with TIGIT-Ig. Clin Immunol. 2019;203:72-80.

\section{Tables}

Due to technical limitations, table 1 and 2 is only available as a download in the Supplemental Files section.

\section{Figures}


$P=0.59$
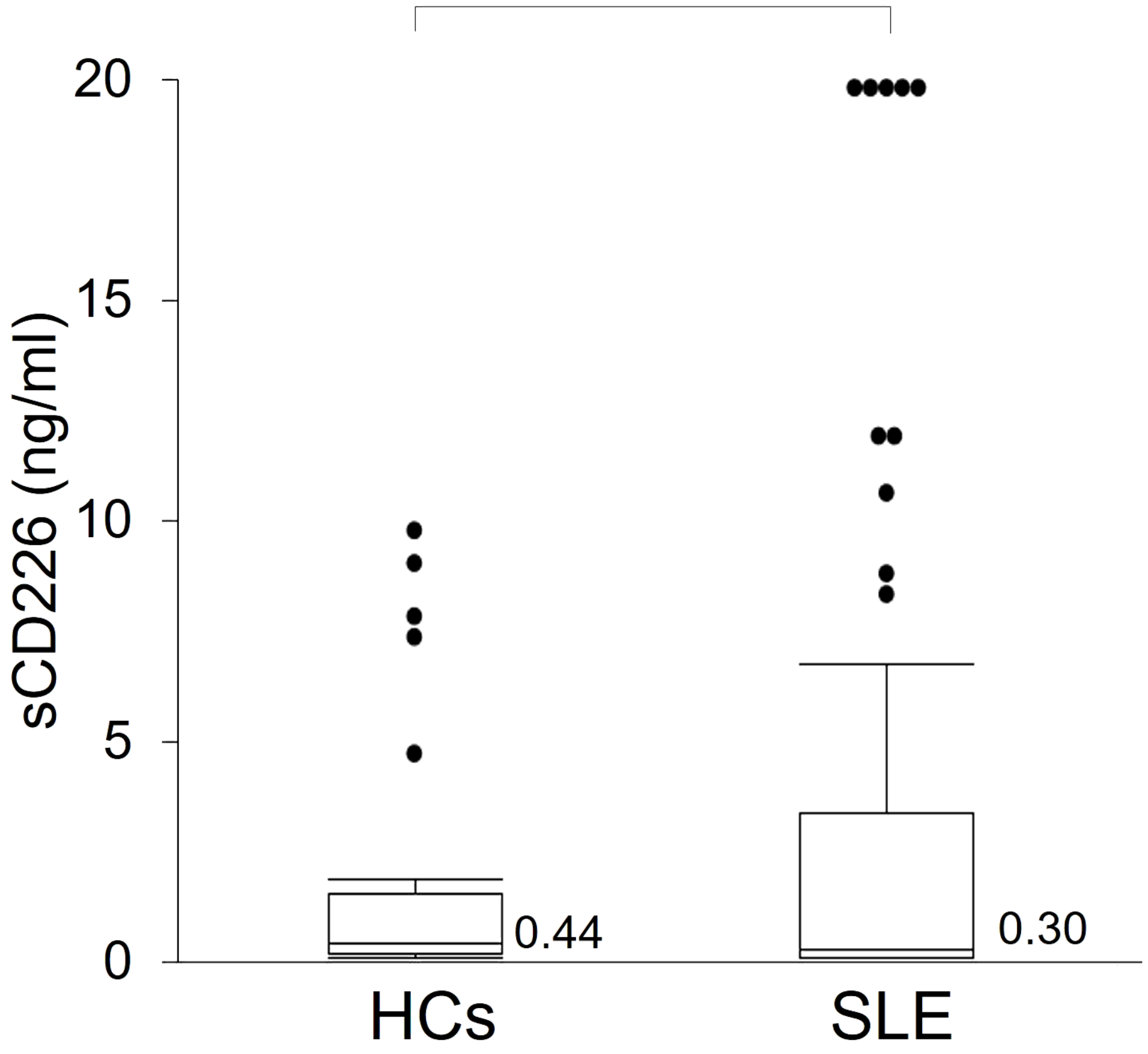

Figure 1

Serum sCD226 levels in SLE patients and HCs Serum SCD226 levels were compared between SLE patients and HCs. Data are shown as box plots. The boxes represent the upper and lower IQRs; lines inside the boxes represent the median; whiskers represent 1.5 times the upper and lower IQRs; points outside the whiskers represent outliers. Statistical differences among groups were evaluated using the 
Mann-Whitney U test. SCD226: soluble CD226; SLE: systemic lupus erythematosus; HCs: healthy controls; IQRs: interquartile ranges.
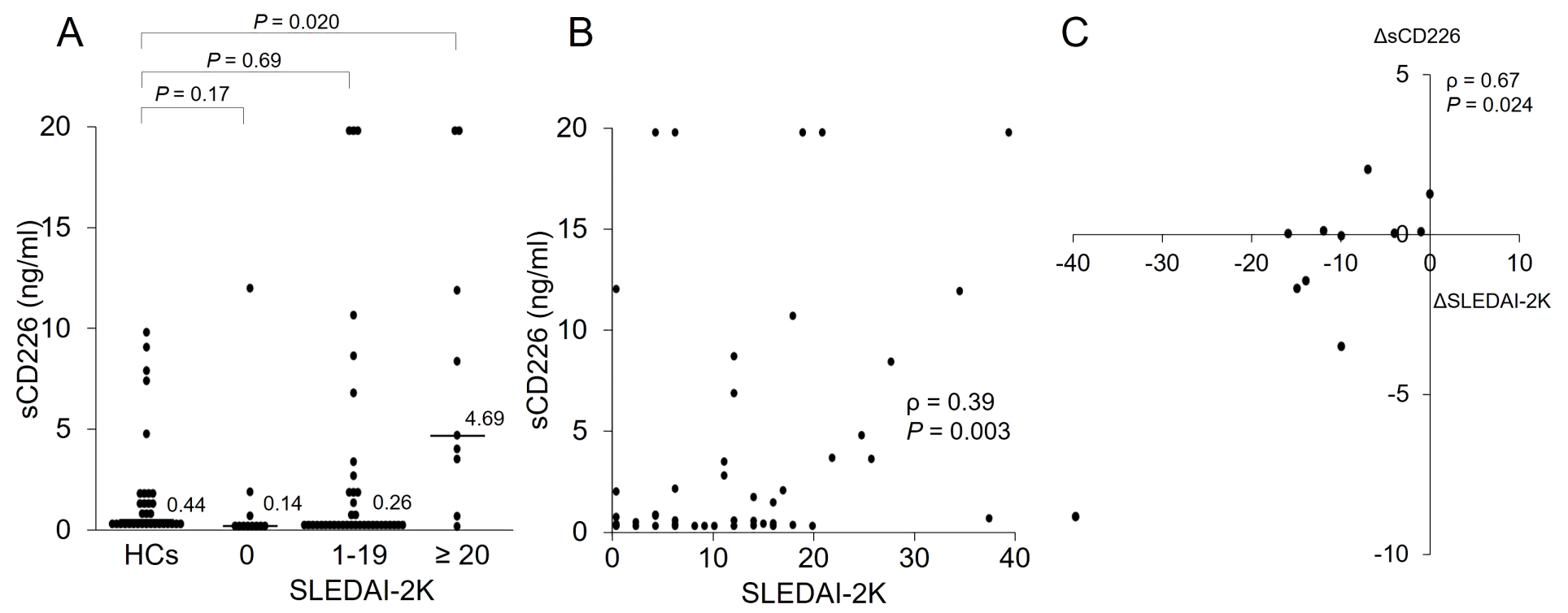

Figure 2

Associations between serum sCD226 levels and SLEDAI-2K scores (A) Serum sCD226 levels were compared between SLE patients with SLEDAI-2K scores of $0,1-19$, and $\geq 20$, as well as HCs. (B) Correlations between serum SCD226 levels and SLEDAI-2K scores in SLE patients. (C) Correlations between the $\triangle S C D 226$ and the $\triangle$ SLEDAI-2K scores in SLE patients. Each data point represents a single subject. Horizontal lines show the median. Statistical differences among groups were evaluated using the Steel test, setting HCs as a control. Correlation analyses were evaluated using Spearman's rank correlation. sCD226: soluble CD226; SLE: systemic lupus erythematosus; SLEDAI-2K: SLE Disease Activity Index 2000; HCs: healthy controls; $\triangle$ SCD226: changes in SCD226; $\triangle$ SLEDAI-2K: changes in SLEDAI-2K. 


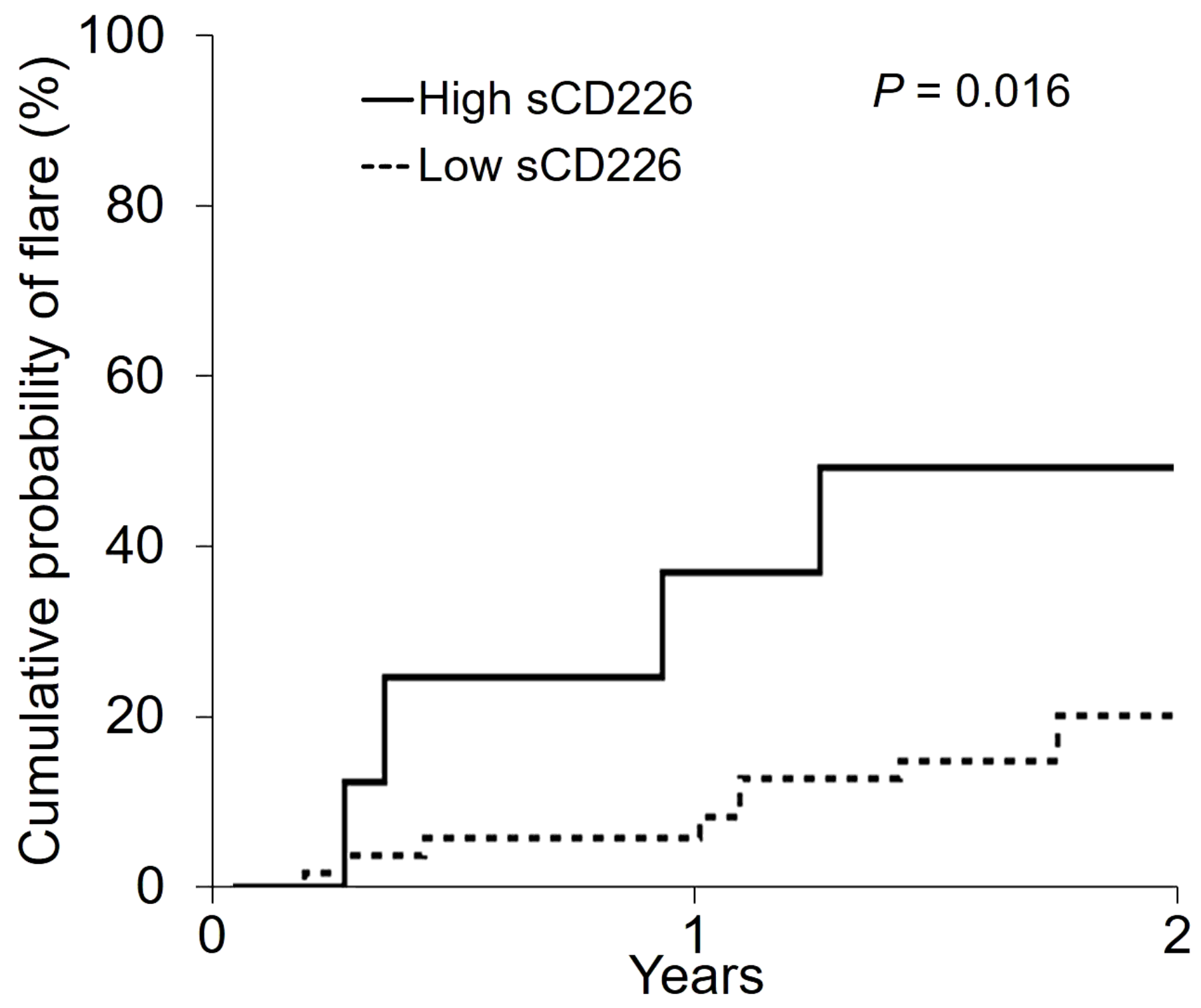

Figure 3

Kaplan-Meier analysis for cumulative probability of flares for patients with different levels of SCD226 Curves were compared using log-rank tests. sCD226: soluble CD226. 

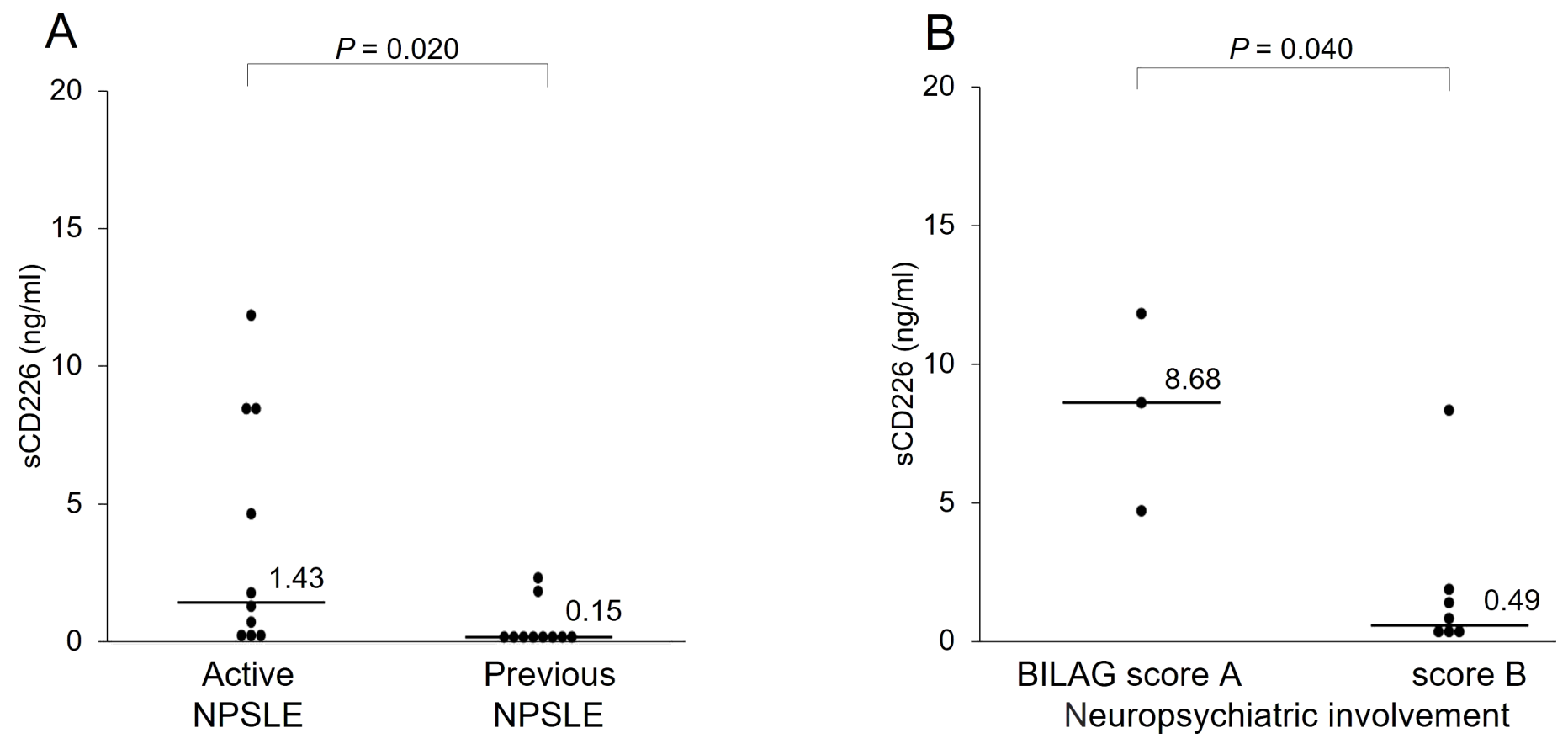

Figure 4

Serum sCD226 levels in SLE patients with neuropsychiatric manifestation (A) Serum sCD226 levels were compared between active NPSLE patients and previous NPSLE patients. (B) Serum sCD226 levels were compared between neuropsychiatric SLE patients with BILAG category A and those with BILAG category B. Each data point represents a single subject. Horizontal lines show the median. Statistical differences among groups were evaluated using the Mann-Whitney U test. SCD226: soluble CD226; SLE: Systemic lupus erythematosus; NPSLE: neuropsychiatric systemic lupus erythematosus; BILAG: British Isles Lupus Assessment Group.

\section{Supplementary Files}

This is a list of supplementary files associated with this preprint. Click to download.

- Tables.docx

- sCD226suppl.pdf 